\title{
Mejora de la resistencia al desgaste abrasivo de un revestimiento Fe-Cr-Mn-C mediante la adición de V
}

\author{
Improvement of the abrasive wear \\ resistance of an Fe-Cr-Mn-C \\ coating through $\mathrm{V}$ addition
}

\author{
Manuel Rodríguez Pérez ${ }^{1}$ \\ Lorenzo Perdomo González ${ }^{1}$, Ismeli Alfonso ${ }^{2}$
}

\footnotetext{
${ }^{1}$ Centro de Investigaciones de Soldadura, Universidad Central "Marta Abreu“ de Las Villas, Carretera a Camajuaní, Santa Clara, Villa Clara, Cuba.

${ }^{2}$ Instituto de Investigaciones en Materiales, Unidad Morelia. Universidad Nacional Autónoma de México, Antigua Carretera a Pátzcuaro, n. 8701, Col. Ex-Hacienda de San José de la Huerta, CEP: 58190, Morelia, Michoacán, México. e-mail:manuelr@uclv.edu.cu, lperdomo@uclv.edu.cu, ialfonso@unam.mx
}

\begin{abstract}
RESUMEN
El presente trabajo persigue incrementar la resistencia al desgaste abrasivo de un revestimiento, aplicado sobre placas de acero al carbono del tipo AISI 1020, utilizando electrodos tubulares revestidos con el sistema de aleación Fe-Cr-Mn-C. Con la finalidad de lograr este incremento, al sistema de aleación se le adicionó V. Los depósitos resultantes se estudiaron mediante microscopía óptica (MO), difracción de rayos-X y ensayos de microdureza. Los ensayos de resistencia al desgaste por abrasión se llevaron a cabo en acuerdo con el procedimiento la norma ASTM G 65-B. Los resultados mostraron que la adición de V al sistema Fe-Cr-Mn-C originó modificaciones microestructurales significativas, disminuyendo la cantidad de martensita en el depósito, y aumentando el tamaño de la zona eutéctica. Asimismo, el V originó la formación de VC, además de los carburos $\mathrm{Cr}_{7} \mathrm{C}_{3}$ presentes para los dos sistemas de aleación. Estas modificaciones microestructurales causaron que los depósitos que utilizan el sistema de aleación Fe-Cr-Mn-V-C presenten una resistencia al desgaste abrasivo significativamente mayor.
\end{abstract}

Palabras clave: Revestimiento, Soldadura, Desgaste, Abrasión, Vanadio, Microestructura.

\begin{abstract}
The aim of this work is to increase the abrasive wear resistance of a coating, applied on plates of AISI 1020 carbon steel, using tubular coated electrodes with the Fe-Cr-Mn-C alloy system. In order to achieve this increase, $\mathrm{V}$ was added to the alloy system. The resulting coating were studied using optical microscopy (MO), $\mathrm{X}$-ray diffraction and microhardness tests. Wear tests were carried out in accordance with the procedure of ASTM G 65-B. Results showed that the addition of V to the Fe-Cr-Mn-C system caused significant microstructural modifications, decreasing the amount of martensite in the coating, and increasing the dimensions of the eutectic zone. Also, $\mathrm{V}$ originated the formation of $\mathrm{VC}$, in addition to the $\mathrm{Cr}_{7} \mathrm{C}_{3}$ carbides present for the two alloy systems. These microstructural modifications provoked that coatings deposited using the $\mathrm{Fe}-\mathrm{Cr}$ $\mathrm{Mn}-\mathrm{V}-\mathrm{C}$ alloy system show a significantly higher abrasive wear resistance.
\end{abstract}

Keywords: Vanadium, coating, welding, abrasive wear, microstructure.

\section{INTRODUCCIÓN}

Para garantizar el desempeño de piezas que trabajan en contacto con minerales, se han desarrollado diversos consumibles de soldadura para la aplicación de revestimientos sobre estas piezas. Entre los sistemas de aleación más empleados para la fabricación de estos consumibles está el $\mathrm{Fe}-\mathrm{Cr}-\mathrm{C}$, cuya microestructura se caracteriza por la presencia de carburos primarios del tipo $\mathrm{Cr}_{7} \mathrm{C}_{3}$ y $\mathrm{Cr}_{23} \mathrm{C}_{6}$ en la zona eutéctica [1,2]. Normalmente poseen un contenido de $\mathrm{C}$ entre 3 y $4 \%$, y de $\mathrm{Cr}$ por encima del $20 \%$. Independientemente de los resultados favorables obtenidos mediante el uso de este sistema de aleación, se han adicionado otros elementos aleantes, 
tales como Mn, W, Nb, V y Mo. Estos elementos favorecen la presencia en la microestructura de carburos de elevada dureza, así como una microestructura con matriz martensítica o austenítica $[1,2,3]$. Los principales carburos reportados son de los tipos $\mathrm{Me}_{7} \mathrm{C}_{3} \mathrm{O} \mathrm{Me}_{23} \mathrm{C}_{6}$, al igual que para el caso del $\mathrm{Cr}$ [4]. En el caso particular de las aleaciones del tipo $\mathrm{Fe}-\mathrm{Cr}-\mathrm{C}$ con adiciones de $\mathrm{Nb}, \mathrm{V}$ y W, puede aumentar la dureza tanto de los carburos primarios como de la matriz. Esto puede ocurrir no solo en la microestructura de las piezas fundidas, sino también en la capa aportada durante el relleno superficial [5,6,7]. No obstante, para lograr una adecuada resistencia al desgaste, es indispensable optimizar la cantidad de elementos de aleación con el fin de garantizar la formación de carburos primarios de alta dureza, además de que tanto estos carburos como la matriz tengan la morfología adecuada. En el caso particular del Mn, este elemento es adicionado a sistemas aleantes $\mathrm{Fe}-\mathrm{Cr}-\mathrm{C}$ con contenidos medios de $\mathrm{Cr}$, no incluyendo otros elementos formadores de carburos [8]. Este sistema es más simple, pero solamente se recomienda en aplicaciones donde el desgaste sea bajo o moderado. Al utilizar este sistema aleante para realizar depósitos mediante electrodos tubulares, se ha obtenido una microestructura caracterizada por el predominio de una matriz austenítica y un eutéctico con la presencia de carburos $\mathrm{Me}_{7} \mathrm{C}_{3}$. También se ha evaluado la resistencia al desgaste abrasivo de electrodos tubulares con la adición de $\mathrm{Nb}$ al sistema de aleación $\mathrm{Fe}-\mathrm{Cr}-\mathrm{C}$ [9]. Los resultados obtenidos muestran un refinamiento de la microestructura de los depósitos y la presencia de carburos $\mathrm{NbC}$ con alta dureza, que repercute en un incremento de la resistencia al desgaste. La presencia de estos microconstituyentes, por su dureza y buena distribución en la matriz, influyen de forma favorable, junto a los carburos $\mathbf{M}_{7} \mathrm{C}_{3}$, en la resistencia al desgaste abrasivo según el estudio realizado en diferentes depósitos de relleno [10].

La existencia en la microestructura de austenita residual a temperatura ambiente se debe a que para contenidos de $\mathrm{C}$ elevados se reduce apreciablemente los valores de inicio de la temperatura de la transformación martensítica [7]. Esta microestructura donde prevalece una matriz austenítica no resulta competente para superficies que están en contacto con partículas abrasivas, siendo necesario: i) una modificación microestructural hacia una matriz donde predominen estructuras aciculares, como podría ser la martensita, y ii) un aumento de la zona eutéctica que presente una mayor dureza debido a la presencia de carburos. Una de las formas posibles de lograr esta transformación podría ser mediante la adición de algún elemento de aleación que contribuya a alcanzar estos dos requisitos, siendo el V una opción viable. Este elemento se ha empleado con resultados satisfactorios en sistemas de aleación recomendados para el relleno superficial mediante los procesos de soldadura, así como en la fabricación de piezas fundidas expuestas al desgaste abrasivo [10,11]. Cuando su contenido en el sistema aleante está en el orden del $5 \%$, el V promueve la formación de carburos en una matriz esencialmente martensítica o austenítica. Por el contrario, cuando el contenido de $\mathrm{V}$ es menor a $5 \%$ se favorece la formación de un eutéctico lebedurítico [7], siendo una microestructura competente en superficies expuestas al desgaste abrasivo. Además, la presencia de $\mathrm{V}$ aumenta la resistencia a la corrosión debido a la elevada afinidad del V por el carbono, lo cual evita la reducción del $\mathrm{Cr}$ en la matriz al formarse menor cantidad de carburos que contienen a este elemento [12]. La adición del V también ha confirmado buenos resultados en aleaciones altamente aleadas al $\mathrm{Cr}$ y Mo, destinadas para piezas expuestas al desgaste abrasivo, debido a que la microestructura alcanza buena ductilidad manteniendo una elevada dureza [13]. Este trabajo obtuvo resultados interesantes respecto a la resistencia al desgaste abrasivo con la presencia de impactos utilizando el $\mathrm{V}$ en el sistema de aleación $\mathrm{Fe}-\mathrm{Cr}-\mathrm{C}-\mathrm{Nb}-\mathrm{V}$ al lograr un excelente balance entre resistencia al desgaste y tenacidad [14]. Se plantea que las propiedades de los depósitos son atribuidas a la presencia de carburos primarios $\mathrm{M}_{3} \mathrm{C}$ finos, ricos en $\mathrm{Nb}$ dispersos en una matriz austenítica, y también por la influencia del $\mathrm{V}$, incluido en los carburos $\mathrm{MC} \mathrm{y} \mathrm{M}_{3} \mathrm{C}$. Una aleación más compleja que toma como base el sistema Fe-Cr-C con adiciones de $\mathrm{Nb}, \mathrm{V}$ y W, fue evaluado en el relleno superficial en cuanto a la resistencia al desgaste abrasivo [6]. Los resultados demostraron que se logra un incremento de la resistencia al desgaste abrasivo por el efecto favorable del $\mathrm{Nb}, \mathrm{V}$ y el $\mathrm{W}$, al precipitar en la microestructura carburos $\mathrm{NbC} \mathrm{y} \mathrm{M}_{7} \mathrm{C}_{3}$ de alta dureza, así como por el efecto de estos elementos de aleación en la tenacidad de la matriz. Tomando en cuenta lo anterior, y debido a la importancia que se le atribuye al V, en el presente trabajo se estudia el efecto de este elemento de aleación sobre las modificaciones microestructurales originadas en revestimientos obtenidos con electrodos tubulares, utilizando el sistema Fe-Cr-Mn-C, así como el efecto de estos cambios sobre la resistencia al desgaste abrasivo de los depósitos.

\section{MATERIALES Y MÉTODOS}

Para el estudio de los depósitos a base de los sistemas Fe-Cr-Mn-C y Fe-Cr-Mn-V-C, desde el punto de vista de la microestructura y de la resistencia al desgaste, se utilizaron 2 variantes experimentales de electrodos tubulares revestidos, de $4 \mathrm{~mm}$ de diámetro, y utilizando como carga aleante ferroaleaciones base $\mathrm{Cr}$ y $\mathrm{Mn}$. El aporte de $\mathrm{V}$ se logra a partir de una aleación obtenida mediante el proceso de aluminotermia aprovechando residuales catalíticos provenientes de la industria química. Estos electrodos fueron fabricados en el Centro de 
Investigaciones de Soldadura de la Universidad Central "Martha Abreu" de Las Villas, Cuba. Se utilizó como revestimiento una mezcla de $50 \%$ de Rutilo $\left(\mathrm{TiO}_{2}\right), 25 \%$ de Fluorita $\left(\mathrm{CaF}_{2}\right), 15 \%$ de Caolín $\left(\mathrm{Al}_{2}\right.$ $\left.\mathrm{Si}_{2} \mathrm{O}_{5}(\mathrm{OH})_{4}\right), 10 \%$ de Calcita $\left(\mathrm{CaCO}_{3}\right)$ y $15 \%$ de Grafito, recubriendo un núcleo tubular fabricado a partir de una cinta de acero AISI 1010. La composición química de los depósitos obtenida con estos electrodos se indica en la Tabla 1. Como puede apreciarse, el contenido de V aportado al depósito es significativo (1.6\%), teniendo en cuenta el efecto de este elemento en los sistemas de aleación, lo que podría garantizar las modificaciones microestructurales deseadas.

Tabla 1: Composición química de los revestimientos experimentales

\begin{tabular}{c|c|c|c|c}
\hline \multirow{2}{*}{ VARIANTES } & \multicolumn{5}{|c}{ COMPOSICIÓN DEL DEPÓSITO (\% EN PESO) } \\
\cline { 2 - 5 } & C & Cr & Mn & V \\
\hline I & 2.3 & 12.5 & 3.8 & - \\
\hline II & 2.4 & 11.9 & 4.0 & 1.6 \\
\hline
\end{tabular}

Estos depósitos se aplicaron mediante soldadura en posición plana, sobre placas de acero AISI 1020 (12 X 100 X $140 \mathrm{~mm}$ ), utilizando el régimen de soldadura que se muestra en la Tabla 2. Para evitar el efecto de la dilución se realizaron depósitos multicapas (5 capas). La temperatura de la placa fue controlada, evitando que la temperatura al inicio de cada pasada fuese superior a los $50{ }^{\circ} \mathrm{C}$. Con esto se logra una velocidad de enfriamiento adecuada para favorecer la transformación isotérmica de la austenita.

Tabla 2: Variables de soldadura para la realización de los depósitos

\begin{tabular}{c|c}
\hline PARÁMETRO & VALORES \\
\hline Tipo de corriente & $\mathrm{CD}+$ \\
\hline Intensidad de Corriente & $100-110 \mathrm{~A}$ \\
\hline Voltaje de arco & $23-26 \mathrm{~V}$ \\
\hline Avance de la soldadura & $3-4 \mathrm{~m} \mathrm{~h}^{-1}$ \\
\hline
\end{tabular}

Para el análisis microestructural de los revestimientos, secciones longitudinales cuadradas $(1.0$ x 1.0 $\mathrm{cm}$ ) de la parte superior de estos se pulieron utilizando técnicas metalográficas convencionales. A continuación, fueron atacados utilizando como reactivo Nital al $2 \%$ durante 15 segundos. Las microestructuras resultantes se observaron mediante un microscopio óptico Neophot 32. Para determinar la microdureza en las diferentes fases del metal depositado se utilizó un microdurómetro marca Shimadzu HMV-2, con una carga de 5.0 N. Esta medición se realizó en las muestras pulidas según los procedimientos anteriormente mencionados, realizando 5 mediciones para cada fase diferente presentes en las secciones longitudinales pulidas. Las fases presentes se estudiaron analizando las secciones longitudinales pulidas, para lo cual se utilizó Difracción de Rayos-X (DRX) mediante un equipo Bruker D-8 Advance, con radiación CuKa operado a 30kV y 25mA. El ensayo de resistencia al desgaste utilizado fue el estandarizado por la norma ASTM G65 [15], empleando los parámetros correspondientes al procedimiento $\mathrm{B}$, establecido cuando el metal aportado por los consumibles a evaluar no forma parte de los sistemas aleantes destinados a la abrasión extrema. Como material abrasivo en los ensayos, se utilizó arena de sílice con un tamaño de partícula entre 150-212 $\mu \mathrm{m}$, empleando los parámetros que se muestran en la Tabla 3.

Tabla 3: Parámetros empleados en el ensayo de resistencia al desgate abrasivo

\begin{tabular}{c|c}
\hline PARÁMETROS & VALORES \\
\hline Flujo de arena & $350 \mathrm{~g} / \mathrm{min}$. \\
\hline Tiempo de ensayo & $10 \mathrm{~min}$. \\
\hline Carga & $130 \mathrm{~N}$ \\
\hline Revoluciones de la banda de goma & $205 \mathrm{rev} . / \mathrm{min}$. \\
\hline Longitud de recorrido & $1400 \mathrm{~m}$ \\
\hline
\end{tabular}

Para cada variante de electrodo se ensayaron 5 probetas obtenidas a partir de placas de acero AISI 1020 realizando 3 pasadas, utilizando los parámetros de soldadura que se mostraron en la Tabla 2. El acabado superficial del área de contacto de las probetas se obtuvo mediante rectificado, cuyas dimensiones fueron de 
25 x 76 × $12 \mathrm{~mm}$. Para determinar la pérdida de metal de las probetas se empleó una balanza digital marca SCALTEC con una precisión de $0.0001 \mathrm{~g}$. Antes de pesar cada probeta, se limpiaron utilizando ultrasonido y etanol.

En la Figura 1a, se muestra el esquema del sistema empleado para el ensayo para evaluar la resistencia al desgaste abrasivo, de acuerdo a lo establecido por la norma ASTM G 65, mientras que en la Figura 1b se muestra el dispositivo utilizado, cuyos parámetros están en correspondencia con los requerimientos que especifica el procedimiento. Este dispositivo fue construido en el Laboratorio de Ensayos Mecánicos de la Facultad de Ingeniería Mecánica de la Universidad Central "Martha Abreu" de Las Villas, Cuba.

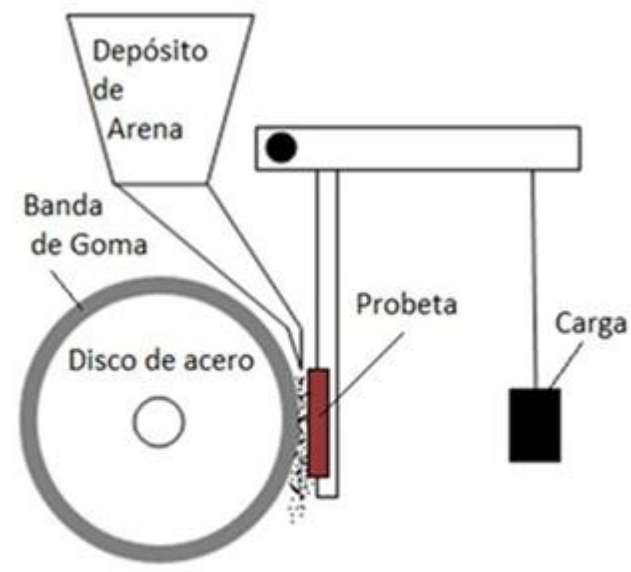

(a)

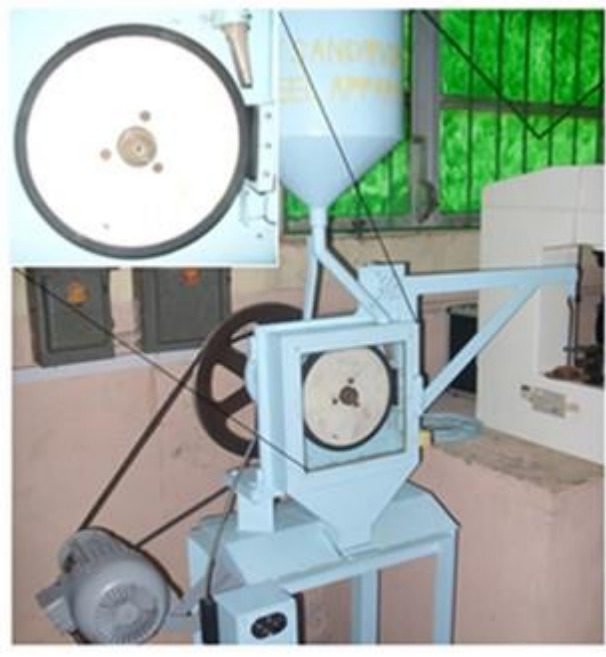

(b)

Figura 1: a) Esquema del ensayo para evaluar la resistencia al desgaste abrasivo de acuerdo a la norma ASTM G65, b) Dispositivo de ensayo utilizado para la determinación de la resistencia al desgaste.

\section{RESULTADOS Y DISCUSIÓN}

\subsection{Análisis microestructural de los revestimientos}

En la Figura 2 pueden apreciarse los difractogramas obtenidos mediante DRX para los revestimientos. Se observa que en ambos casos los picos más intensos están situados en valores de 2 de 43.5 y $44.9^{\circ}$, correspondiendo a la la austenita o cementita, y a la martensita, respectivamente. También se observan picos correspondientes a carburos $\mathrm{Cr}_{7} \mathrm{C}_{3}$. Su tamaño reducido se debe a que esta fase presenta los picos más intensos en la misma ubicación que los picos más intensos de las fases mayoritarias, siendo entonces solo visibles picos secundarios. Para el caso de los depósitos donde se añade $1.6 \%$ de V también se observó la presencia de VC. Estos picos son pequeños debido al bajo contenido de esta fase, lo cual está determinado por la cantidad de $\mathrm{V}$ en el sistema. Otro cambio significativo que se aprecia es la disminución de la altura de los picos de martensita para el caso de los depósitos donde se utilizó $\mathrm{V}$, lo cual indica que la cantidad de esta fase es menor para este caso. Asimismo, al adicionar $\mathrm{V}$ aumentó la altura de los picos de austenita y cementita, lo cual indica el aumento de la cantidad de estas fases. 


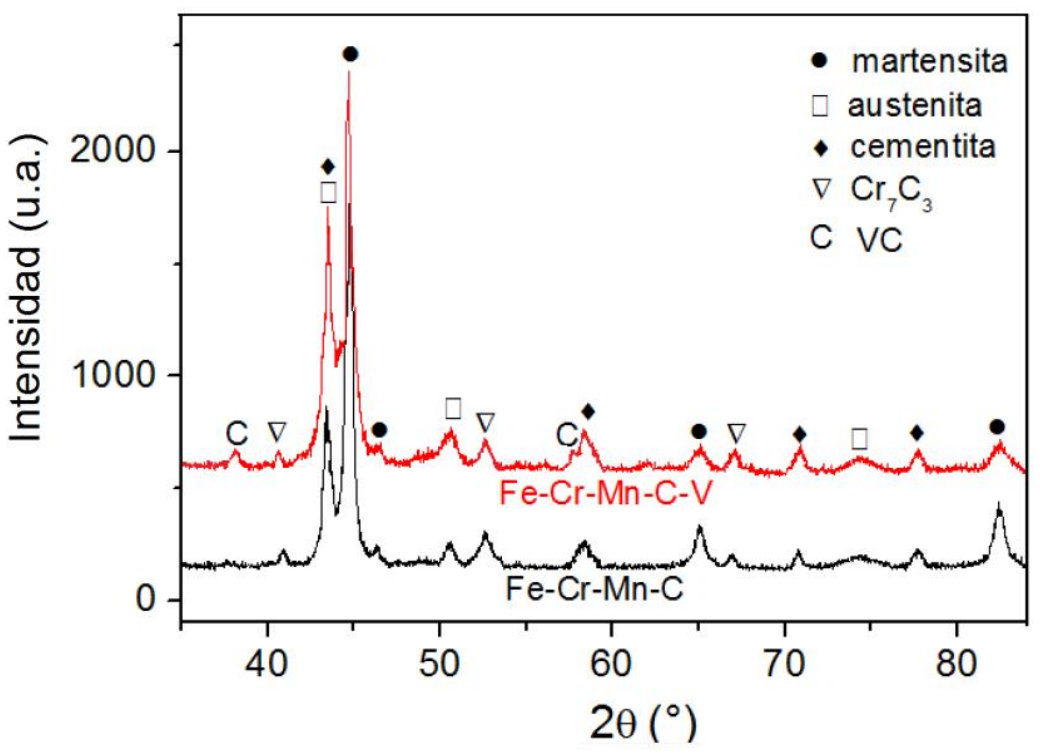

Figura 2: Difractogramas de los depósitos obtenidos con los sistemas Fe-Cr-Mn-C con y sin V.

En las Figuras 3a y 3b se pueden observar las micrografías obtenidas mediante microscopia óptica de los depósitos realizados con los electrodos de las variantes I y II, respectivamente. Como se puede apreciar, en la microestructura de ambos depósitos se distinguen una zona oscura y otra clara, con una morfología similar. La fase predominante es la martensita (zona oscura), mientras que el eutéctico presenta un contenido menor (zona clara). Esta zona eutéctica está constituida por cementita, austenita y los carburos antes mencionados, lo cual se corresponde con lo obtenido mediante DRX. Utilizando el software SigmaScan Pro 5.0, se determinó el área ocupada por las fases en las microestructuras de los depósitos. Los resultados arrojan que para el caso de la variante I la martensita ocupa el $75 \%$ y la zona eutéctica un $25 \%$, mientras que para la variante II, esta relación alcanzó valores de 64 y $36 \%$. En las Figuras 3c y 3d, que son imágenes ya procesadas, puede apreciarse de manera más notable el contenido de estas fases. Estos resultados explican las diferentes intensidades de los picos obtenidos mediante DRX para los revestimientos con y sin V. Este elemento influye de manera significativa en la formación de determinadas fases como son la cementita y el eutéctico, modificando la morfología de las microestructuras de los depósitos de relleno [5,6,7] , lo cual se corresponde con los depósitos realizados con el electrodo de la variante II, que aporta $1.6 \%$ de V. Igualmente el V origina que aumente apreciablemente la temperatura de la transformación eutéctica, disminuyendo su valor entre los diagramas de fases estable y metaestable [16]. La presencia de la austenita retenida en el eutéctico de la microestructura en ambos casos, se debe fundamentalmente al efecto que ejerce el $\mathrm{Mn}$ en la estabilidad de esta fase formada a partir de la reacción eutéctica [17,18]. Para las fundiciones blancas hipoeutécticas con una composición similar de C a la de los depósitos de los electrodos de las variantes I y II (2.3 y 2.4 \%), al solidificar se pueden formar diferentes fases en las microestructuras, lo cual depende de la velocidad de enfriamiento y el posible efecto de elementos de aleación adicionales [16,19]. Los depósitos realizados con los electrodos de las variantes I y II se desarrollaron sin precalentamiento del metal base, lo que provocó una velocidad de enfriamiento apreciable, superior a las consideradas en los diagramas metaestables. Esta velocidad de enfriamiento provocó que la austenita proeutéctica de forma dendrítica, que se forma al solidificar los depósitos, se haya transformado en una matriz martensítica (como se pudo observar en las micrografías de la Figura 3a-b. En este caso, al disminuir el tiempo de enfriamiento, un determinado contenido de C permanece en solución solida en la austenita, fenómeno que provoca que ésta se transforme finalmente en martensita y no en perlita, como ocurre cuando las aleaciones se enfrían lentamente [20]. También estas dos variantes de electrodos aportan $\mathrm{Cr}$ y $\mathrm{Mn}$, los cuales tienen influencia significativa en la microestructura final de los depósitos. Debido a que el contenido de $\mathrm{Cr}$ es superior al $2 \%$, no se forma cementita aleada $\left(\mathrm{Me}_{3} \mathrm{C}\right)$, debido a que se superan los límites de saturación de ésta por dicho elemento y aparecen entonces diferentes tipos de carburos de cromo [20,21]. Considerando la relación $\mathrm{Cr} / \mathrm{C}$ en la microestructura del metal depositado con los electrodos I y II, se forma principalmente carburos $\mathrm{Cr}_{7} \mathrm{C}_{3}$ que se manifiestan en la zona eutéctica [22,23].

Por otra parte, la formación de estos carburos a partir de la temperatura eutéctica trae como resultado la disminución de la cantidad de $\mathrm{C}$ en solución solida de la austenita metaestable, fenómeno que eleva la temperatura de inicio de la transformación martensítica (Ms) [19,20]. Lo anterior es otra de las causas que favorece que en la microestructura de la capa de relleno con los consumibles evaluados se forme finalmente una matriz martensítica. El Mn, que está en la composición química de ambos depósitos en una cantidad en el 
orden del $4 \%$, es un fuerte estabilizador de carburos, aunque no es un fuerte formador de estos [17]. Este efecto del Mn sobre la estabilidad de los carburos que se forman en la zona eutéctica indirectamente origina que disminuya la cantidad de $\mathrm{C}$ en solución solida en la austenita, y por tanto también aumenta la temperatura de la transformación isotérmica de esta fase. También el Mn para los contenidos correspondientes a los depósitos de los electrodos de las variantes I y II aumenta la templabilidad al reducir la velocidad crítica necesaria para que ocurra la transformación isotérmica de la austenita [1,16,20]. El Mn igualmente contribuye a la presencia de martensita en la matriz de la microestructura en la capa de relleno con los dos electrodos evaluados. Por otra parte, el V es considerado como uno de los elementos de aleación con mayores potencialidades para la formación de carburos [7,13]. Esto contribuye a que en la zona eutéctica de la microestructura obtenida con el electrodo de la variante II, además de los carburos $\mathrm{Cr}_{7} \mathrm{C}_{3}$, estén presentes carburos VC, lo cual ya se analizó mediante DRX.

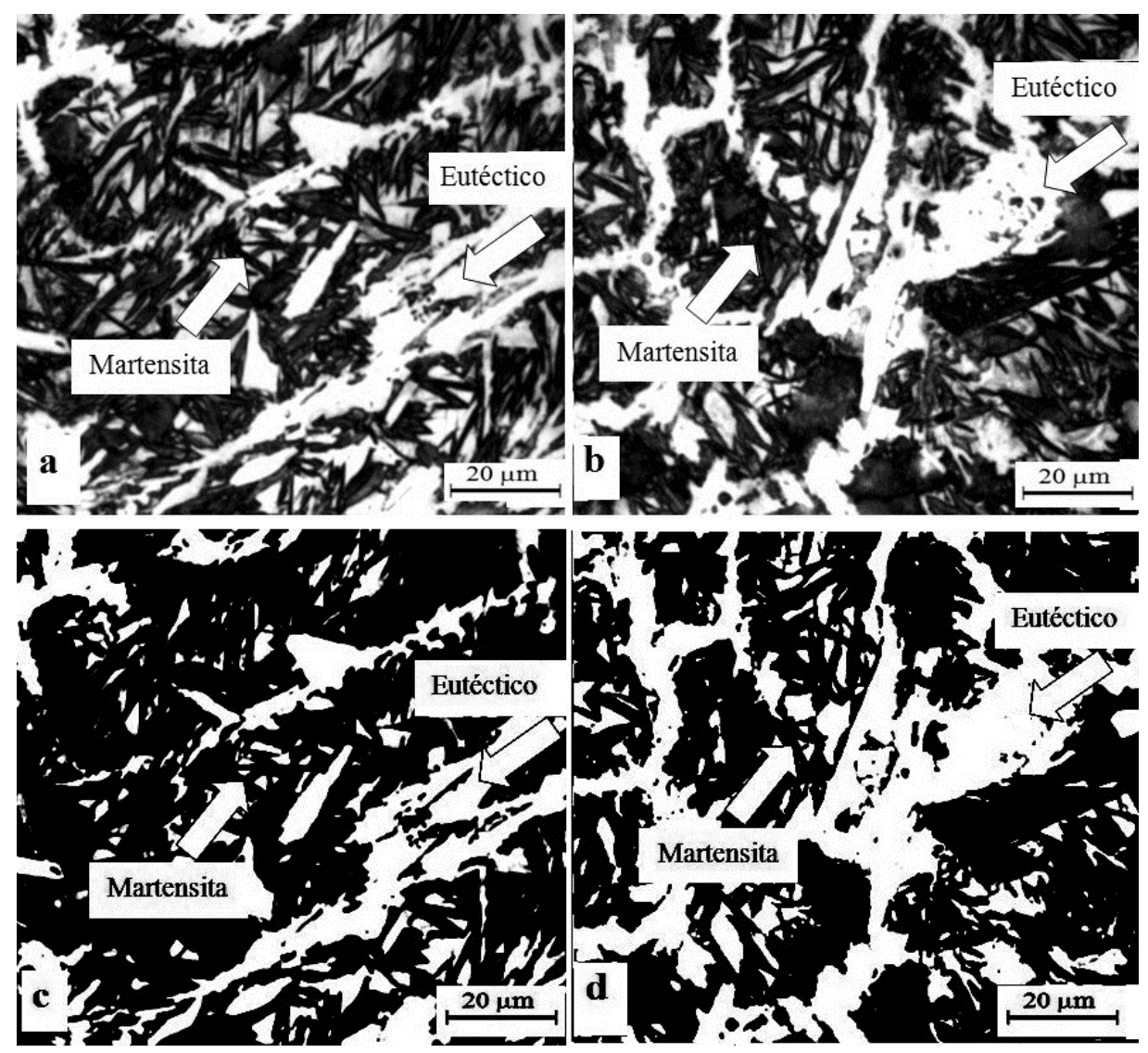

Figura 3: Microestructura obtenida mediante microscopia óptica para los depósitos aplicados utilizando los electrodos de las variantes I (a), y II (b), e imágenes procesadas correspondientes a estas micrografías (c y d).

Lo anteriormente explicado en relación con la cantidad de fases y los carburos de $\mathrm{V}$ justifica los resultados de las microdurezas obtenidas, como se aprecia en la Tabla 4, siendo significativamente superior la dureza de la zona eutéctica para el depósito que contiene V. Los carburos de V son los causantes de este incremento. En la matriz martensítica los resultados fueron similares.

Tabla 4: Resultados de la microdureza en la microestructura obtenida en los depósitos con electrodos tubulares revestidos utilizando los dos sistemas aleantes.

\begin{tabular}{c|c|c}
\hline \multirow{2}{*}{ VARIANTES } & \multicolumn{2}{|c}{ MICRODUREZA (HV) } \\
\cline { 2 - 3 } & MATRIZ & EUTÉCTICO \\
\hline I & 533 & 846 \\
\hline II & 516 & 915 \\
\hline
\end{tabular}




\subsection{Comportamiento de la resistencia al desgaste del metal depositado}

Para evaluar el comportamiento de la resistencia al desgaste del metal depositado por los consumibles de las variantes I y II, se determinó inicialmente la pérdida de masa de cada una de las probetas después de ser sometidas a las condiciones de ensayo anteriormente descritas. A partir de estos datos, las pérdidas de volumen se determinaron por medio de la ecuación 1.

$$
\text { Pérdida de volumen }\left(\mathrm{mm}^{3}\right)=\frac{\text { masa pérdida }(\mathrm{g})}{\text { densidad }\left(\mathrm{g} / \mathrm{cm}^{3}\right)} \times 1000
$$

En la Tabla 5, aparecen las pérdidas de masa y volumen de cada una de las muestras evaluadas, en correspondencia a lo establecido por la norma ASTM G65. Estos valores se procesaron estadísticamente con la finalidad de determinar las diferencias que existen entre ellos, lo cual se mostrará más adelante.

Tabla 5: Resultados de la pérdida de masa (en $\mathrm{g}$ ) y de volumen (en $\mathrm{mm}^{3}$ ) para las muestras ensayadas.

\begin{tabular}{|c|c|c|c|c|c|}
\hline \multicolumn{2}{|c|}{ PROBETAS } & \multirow{2}{*}{$\begin{array}{l}\text { PESO ANTES } \\
\text { DEL ENSAYO }\end{array}$} & \multirow{2}{*}{$\begin{array}{l}\text { PESO DESPUÉS } \\
\text { DEL ENSAYO }\end{array}$} & \multirow{2}{*}{$\begin{array}{c}\text { DIFERENCIA } \\
\text { DE PESO }\end{array}$} & \multirow{2}{*}{$\begin{array}{l}\text { PÉRDIDA DE } \\
\text { VOLUMEN }\end{array}$} \\
\hline VARIANTE & CÓDIGO & & & & \\
\hline \multirow{5}{*}{ I } & A & 91,2487 & 91,1457 & 0,1040 & 13,345 \\
\hline & B & 89,8561 & 89,7478 & 0,1113 & 14,272 \\
\hline & $\mathrm{C}$ & 89,6927 & 89,6043 & 0,0884 & 11,334 \\
\hline & $\mathrm{D}$ & 87,5678 & 87,4829 & 0,0849 & 10,389 \\
\hline & $\mathrm{E}$ & 90,1234 & 90,0029 & 0,1205 & 15,456 \\
\hline \multirow{5}{*}{ II } & $\mathrm{F}$ & 92,2743 & 92,2022 & 0,0720 & 9,234 \\
\hline & G & 88,1219 & 88,0343 & 0,0876 & 11,231 \\
\hline & $\mathrm{H}$ & 88,0763 & 87,9939 & 0,0824 & 10,567 \\
\hline & I & 92,1567 & 92,0917 & 0,0650 & 8,345 \\
\hline & $\mathrm{J}$ & 86,2312 & 86,1552 & 0,0760 & 11,233 \\
\hline
\end{tabular}

Para comparar desde el punto de vista estadístico la resistencia del metal depositado con las variantes de electrodos I y II, se realizó el análisis teniendo en cuenta la pérdida de volumen, empleando el programa Statgraphics Centurion XV. La comparación de los valores medios de la pérdida de volumen se puede apreciar en la Figura 4. Los resultados indican que hay una diferencia estadísticamente significativa al comparar la pérdida de volumen en las muestras ensayadas del metal depositado con las variantes de electrodos I y II, siendo mayores los volúmenes perdidos para el caso en el que no se adicionó V. Lo anterior significa que el metal depositado con el electrodo de la variante II (Fe-Cr-Mn-V-C) tiene mayor resistencia al desgaste abrasivo, lo que se puede explicar debido al incremento del área superficial que ocupa la zona eutéctica para esta variante, así como por la mayor dureza de esta zona $(915 \mathrm{HV})$ en comparación con la medida para la misma zona de la variante I ( $846 \mathrm{HV})$. El aumento de la zona eutéctica y su dureza para la microestructura del metal depositado con los electrodos de la variante II se debe al aporte de $1.6 \%$ de V, cuyo efecto fue explicado anteriormente. Aunque la resistencia al desgaste abrasivo no es proporcional a la dureza, las fases presentes y su distribución juegan un papel determinante.

Aunque la resistencia al desgaste abrasivo no es proporcional a la dureza, las fases presentes y su distribución sí juegan un papel determinante. En la microestructura del metal depositado con los electrodos de la variante II, que aporta $1.6 \%$ de V, están presentes los carburos VC, con un efecto significativo en el aumento de la resistencia al desgaste abrasivo. La presencia de esta fase en la zona eutéctica debido a que poseen una dureza en el orden de $2500 \mathrm{HV}$ [6], reducen el efecto de desbaste de las partículas abrasivas, ya que contribuyen junto a los demás carburos a bloquear los microcortes y por tanto, hacen que aumente la resistencia al desgaste $[5,24]$ 


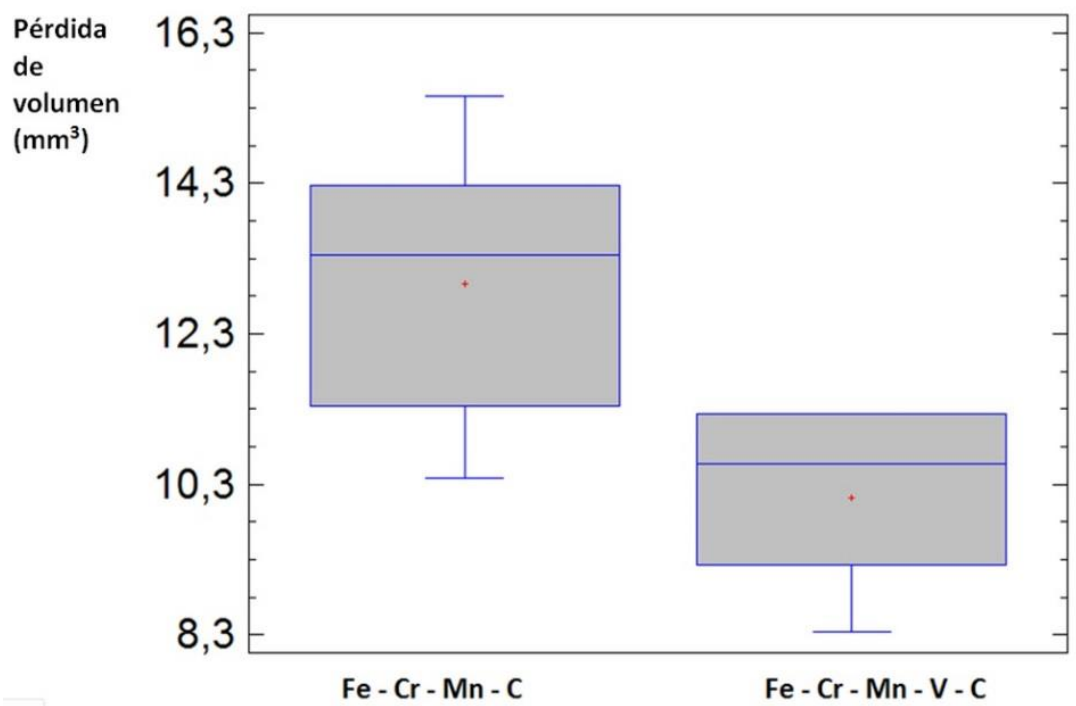

Figura 4: Comparación de la pérdida de volumen de las muestras ensayadas obtenidas con los electrodos de las variantes I y II.

Una forma de corroborar el comportamiento al desgaste es el estudio de las superficies de las muestras una vez concluido el ensayo. Por tal motivo las superficies desgastadas se observaron mediante MO, para así poder comparar el daño provocado por las partículas abrasivas. En las Figuras 5a y 5b se aprecian imágenes obtenidas por MO de estas superficies para los depósitos obtenidos utilizando las variantes I y II, respectivamente. Para los depósitos realizados con el electrodo de la variante I (Figura 5a), se aprecia un mayor efecto del abrasivo según se manifiesta por el mayor ancho de las huellas, que son provocadas por los microcortes de las partículas abrasivas. Este mayor ancho de las huellas se puede relacionar con una mayor pérdida de volumen de metal, en correspondencia con lo observado en la Figura 4. Con la finalidad de analizar cuantitativamente las diferencias entre estas dos superficies sometidas a abrasión, se determinaron sus dimensiones fractales $\left(d_{f}\right)$. Este parámetro ha sido utilizado para comparar superficies desgastadas [25], ya que $d_{f}$ se relaciona con la complejidad de una estructura o geometría: mientras mayor es $d_{f}$, más heterogénea y compleja es el objeto. Por tal motivo, la determinación de este parámetro a partir de imágenes sería un complemento a las mediciones de las pérdidas de material. En este caso en particular, a medida que $d_{f}$ aumenta la estructura desgastada es más compleja, significando que el desgaste es mayor. $d_{f}$ se determina de manera muy sencilla utilizando el método de conteo de cajas a partir de imágenes. Los valores medidos de $d_{f}$ utilizando este método mediante el programa Image J [26] para los revestimientos I y II fueron 1.89 y 1.76, respectivamente, lo que comprobó el mayor desgaste para el revestimiento aplicado con el sistema sin V (revestimiento I).

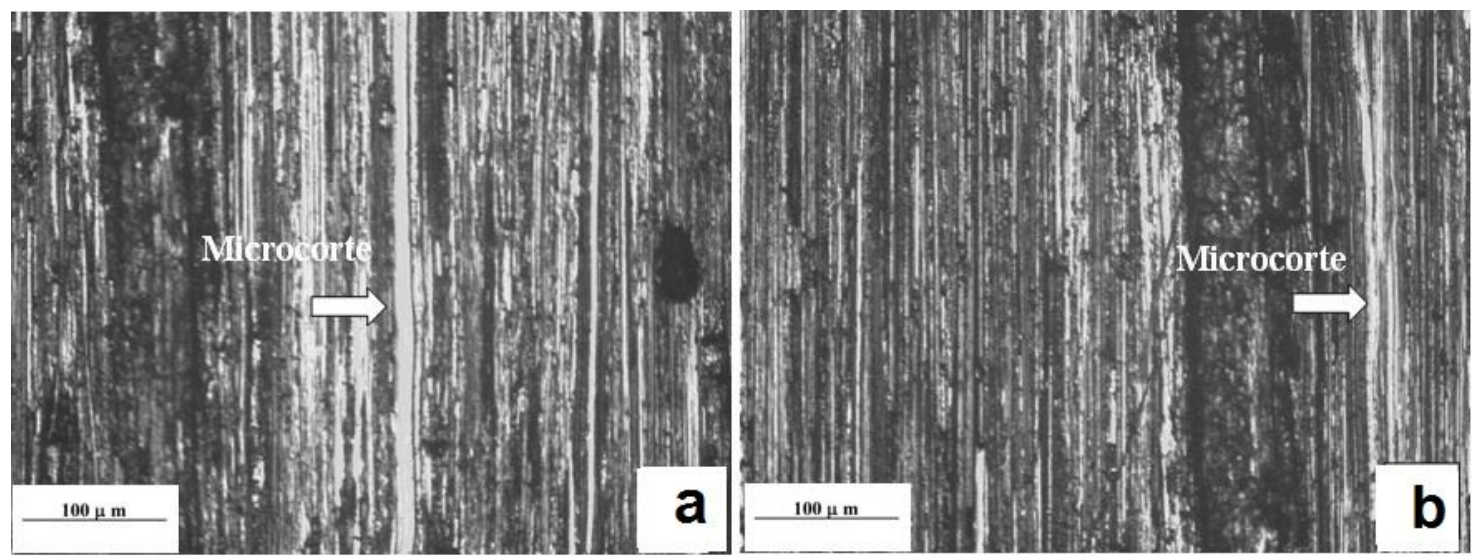

Figura 5: Huellas originadas por los microcortes durante los ensayos de desgaste para las probetas correspondientes a los electrodos de las variantes I (a), y II (b). 


\section{CONCLUSIONES}

1. Las microestructuras de los revestimientos obtenidos utilizando los sistemas Fe-Cr-Mn-C y Fe-Cr$\mathrm{Mn}-\mathrm{C}-\mathrm{V}$ son similares, estando formadas por una matriz martensítica y eutéctico. Para ambos revestimientos se detectó la formación de $\mathrm{Cr}_{7} \mathrm{C}_{3}$.

2. La adición de $\mathrm{V}$ originó el aumento del tamaño de la zona eutéctica y la formación de carburos VC, disminuyendo el contenido de la matriz martensítica.

3. La dureza y el área ocupada por la zona eutéctica aumentó para el sistema Fe-Cr-Mn-C-V debido a la formación de los carburos VC. La dureza de la matriz martensítica no presentó cambios significativos.

4. Al adicionar $\mathrm{V}$ se logró aumentar de manera significativa la resistencia al desgaste abrasivo de los revestimientos aplicados a partir del sistema Fe-Cr-Mn-C.

5. El estudio fractal de las superficies sometidas a desgaste fue usado con éxito como una herramienta complementaria para analizar comparativamente el desgaste de los materiales experimentales.

\section{AGRADECIMIENTOS}

Los Autores agradecen al Instituto de Investigaciones de Materiales de la Universidad Autónoma de México por el apoyo en relación a la disponibilidad de las instalaciones para desarrollar la Investigación. Además, se agradece a la Universidad Nacional Autónoma de México por el apoyo al Dr. Manuel Rodríguez Pérez para realizar una estancia de investigación en el Instituto de Investigaciones en Materiales, Unidad Morelia, de la UNAM, a través del programa PREI de la DGAPA.

\section{BIBLIOGRAFÍA}

[1] KOTECKI, D., "Abrasion resistance of iron based hardfacing alloys", Welding Journal, Welding Research Supplent, August., pp. 269 - 278, 1995.

[2] RODA, C., LOUREIRO, A., PITA, J., "Comportamiento frente al desgaste abrasivo de las aleaciones con tendencia a la formación de carburos aplicados por soldadura", Mantenimiento, v. 134, pp. 78 - 89, 2000.

[3] CHATTERJEE, S., PAL, T.K., "Wear behavior of hardfacing deposits on cast iron", Wear, v. 255, pp. 417-425, 2003.

[4] MATSUBARA, Y., SASAGURI, N., "Solidification and Abrasion Wear of White Cast Irons alloyed with $20 \%$ Carbide forming Elements”, Wear, v. 250, pp. 502-510, 2001.

[5] FARUK, U., ANDRIJA P., "The effect of Vanadium content on the properties of Fe-C-Cr-V white Cast Iron”, Materiali in Tehnologije. v. 37, pp.19-24, 2003.

[6] WANG, Q., LI, X., "Effects of Nb, V, and W on Microstructure and Abrasion Resistance of Fe-Cr-C Hardfacing Alloys”, Welding Journal, v. 89, pp. 134-139, 2010.

[7] MIRJANA, F., KAMBEROVIC, Z., KORAC M., "Solidification of High Chromium White Cast Iron with Vanadium”, Materials Transactions, v. 52, n. 3, pp. 386 -390, 2011.

[8] MOLERIO, D., "Electrodo tubular revestido para el recubrimiento superficial de los martillos y piezas de los molinos empleados en la trituración de sal", Disertación MSc, Universidad Central "Marta Abreu" de Las Villas, 2011.

[9] CRUZ CRESPO, A., FERNÁNDEZ-FUENTES, R., VALTAIR FERRARESSI, A., "Microstructure and Abrasion Resistance of Fe-Cr-C and Fe-Cr-C-Nb Hardfacing Alloys Deposited by S-FCAW and Cold Solid Wires”, Soldagem \& Inspeção, v.23, n.3, pp. 342-353, 2016.

[10] SINHA, A.K., Ferrous Physical Metallurgy. Butterworth Publisher, Stoneham, Butterworth Publishers, EE. UU, 1989.

[11] RADULOVIC, M., "The Influence of Vanadium on the Microstructure and Mechanical Properties of a High Chromium White Iron”, Thesis Ph.D , University of Belgrade, Belgrade, 1991.

[12] JIU, C., "High Chromium Cast Iron and Its Application", Metallurgical Transactions, v.16A, pp.10-20, 1999.

[13] TODIC, A., "Influence of Vanadium on Mechanical Characteristics of Air - Hardening Steel", FME Transactions, n. 39, pp. 49-54, 2011. 
[14] CORREA, E.O., ALCANTARA, N. G., TECCO, D.G., et al., "The relationship between the Microstructure and Abrasive Resistance of a Hardfacing Alloys in the Fe-Cr-C-Nb-V System", Metallurgical and Materials Transactions, v. 38, pp. 1671-1680, 2007.

[15] Norma ASTM G65, "Standard Test Method for Measuring Abrasion Using the Dry Sand/Rubber Wheel Apparatus", 2001.

[16] DAVIS, J.R., "Classification and Basic Metallurgy of Cast Irons”, ASM Specialty Handbook Cast Iron. pp. 4-7,1996.

[17] HIGUEREA, F., MORENO, M., SUAREZ B., "Evolución microestructural del acero austenítico al manganeso sometido a tratamiento térmico de temple y revenido", Revistas Ingenierías, Universidad de Medellin, v. 9, n. 17, pp.95-206, 2010.

[18] VISHAL, I., MENGHANI, J.V., CHANNIWALA, S.A., "Studies on the effect of element in iron base hardfacing alloys", Transactions of the Indian Institute of Metals, v. 67, n. 3, pp. 343-349, 2013.

[19] GUNDLACH, R.B., "Influence of abrasive hardness on the wear resistance of high chrome", Wear, Lausanne. v. 46, n. 1, pp.77-108, 1978.

[20] FILIPOVIC, Z., KAMBEROVIĆ, Ž., KORAĆ, M., "Effect of heat treatment on toughness and wear resistance of Fe-C-Cr-Nb alloys", Journal of Metallurgy, v. 14, n. 4, pp. 243-252, 2008.

[21] GOYOS, L., "Influencia de la microestructura en la resistencia al desgaste de los aceros al cromo", Revista Construcción de Maquinarias, v. 11, n.1, pp. 44-49, 1986.

[22] ATAMERT, S., BHADESHIA, H.K., "Microestructure and stability of Fe-Cr-C hardfacing alloys", $M a-$ terials Science and Engineering, v. 130, pp. 101-111, 1990.

[23] CHE, W. K., Chieh, F., Sheng, H.W, et al., "Microstructure and Wear Characteristics of Hypoeutectic, Eutectic and Hypereutectic $(\mathrm{CrFe})_{23} \mathrm{C}_{6}$ carbides in Hardfacing Alloys", Materials Transactions, v. 48, n. 9 , pp. 2324- 2328, 2007.

[24] BUCHELY, M.F., GUTIÉRREZ, J.C., LEÓN, L.M., TORO, A. "The effect of microstructure on abrasive wear of hardfacing alloys", Wear, v.48, n. 3, pp. 52-61, 2005.

[25] PANIN, A.V. LYAZGIN, A.O., SHESTERIKOV, E.V., et al., "Fractal Analysis of the Evolution of Friction Surfaces of Galvanic AuNi Coatings”, Technical Physics Letters, v. 38, n. 5, pp. 484-487, 2012.

[26] RASBAND W.S. IMAGEJ, U. S. National Institutes of Health, Bethesda, Maryland, USA, http://imagej.nih.gov/ij/, 1997-2015.

\section{ORCID}

Manuel Rodríguez Pérez

https://orcid.org/0000-0002-4092-3470

Lorenzo Perdomo González https://orcid.org/0000-0002-3425-1487

Ismeli Alfonso

https://orcid.org/0000-0001-7311-8614 